\title{
Requirements Management Model (RMM): A Proposed Model for Successful Delivery of Software Projects
}

\author{
Muhammad Yaseen \\ Engineering Research \& IT \\ Services Provider Pvt Ltd, \\ Peshawar, Pakistan
}

\author{
Zahid Ali \\ Engineering Research \& IT \\ Services Provider Pvt Ltd, \\ Peshawar, Pakistan
}

\author{
Muhammad Humayoun Khan \\ Xinjiang University, Urumqi, \\ Xinjiang, People's Republic of \\ China (PRC),
}

\begin{abstract}
Requirements management is not easy task especially in global software development where clients and vendors are far away from each other's. For successful implementation of any software project, proper and efficient requirements management is necessary. Purpose of this current work is development of requirements management model (RMM) that can address the factors that have positive impact and the factors that have negative impact during management of requirements.
\end{abstract}

The propose model will provide solutions to the challenges during requirement management. Systematic literature review (SLR) and empirical research study will be conducted for achieving the goals and objectives. The expected results of this study will be RMM that will help vendor organizations in better management of software requirements.

\section{Keywords}

Systematic literature review, requirement engineering, requirement management model, software requirements.

\section{INTRODUCTION}

Requirement engineering (RE) is the systematic and discipline way of collecting user requirements for a software system and to manage it [1]. RE consists of the following phases;

- Requirement elicitation: In this phase, user requirements and functional requirements for a software system are collected. Requirements from users are collected by applying various elicitation techniques such as background study, interview, prototyping, questionnaire, apprenticing, brainstorming etc.

- Requirement analysis: In this phase, the collected functional requirements are analysed and examined in order to purify it before further processing. Requirements are analysed using models and that's why analysis phase is also known as requirements modelling. Four types of models are normally used for analysis. First type of model such as context model analyse requirements from external perspective. Second type of model such as USE CASE model analyse requirements from user perspective. Third type of model analyse requirements from internal perspective such as class diagrams and fourth type of model analyse requirements from behavioural perspective.

- Requirements specification: In this phase all requirements are specified in the form of document known as software requirements specification (SRS) document. The quality of SRS is totally dependent on requirements analysis and elicitation phase which indicates the significance of elicitation phase. The success of software product depends on how well requirements are implemented which shows the importance of RE phase of software development life cycle.

- $\quad$ Requirements management: In parallel of above all phases, role of requirements management is also very important. The success implementation of all phases is associated with effective requirements management. Requirements management is not limited to these tasks only but it also deals with other activities such as how requirements should be collected and what type of tools will be used for it, how requirements should be prioritized, how requirements will be divided on parallel development team members, how implemented requirements should be integrated, how much time and cost will be required for the implementation of software requirements. All these questions are associated with requirements management.

There is significance difference between project management and requirements management. Project management deals with all perspectives of project and is quite broad term as compare to requirements management where the scope is limited to requirements only.

Although enough work has been done in this field but no such model is developed which can address all possible success factors and challenges during managing requirements so that software projects can be delivered on time to clients. According to many authors, timely delivery of requirements within specified budget is considered to be big success factor and this can be assured only if requirements are well managed. Almost more than 50\% of projects failure reason is bad management of requirements.

The objective of this research study is to develop RMM in order to support vendor organizations in better management of requirements. This model will address challenges and success factors during requirement management. The propose model will address the solutions and practices needed to better implement success factors during management and to reduce the effect of the challenges. For achieving the objectives, we will do the Systematic Literature Review (SLR) and empirical study. For the implementation of factors, practices and solutions will be extracted through SLR and then questionnaire survey will be conducted to validate success factors, challenges and practices. Survey will be conducted in software industry to mention some new practices not mentioned in literature before. After finding the practices and solutions the propose model will be developed. The aim is to reduce the gap between researchers and software development vendors. Other researchers also adopted the same methodology in other fields to suggest such models [14]. 


\section{BACKGROUND STUDY}

According to [2], there are three big factors that become the basis for software project failure. First factor is that project doesn't meet all the functionalities according to SRS. Second factor is time and third is cost i.e. the project is not delivered in time and in defined budget. This means for success of any project, requirements management plays an important role.

According to [3], the failure of most of the projects is traced to its lack of effective requirement management activities. The failure of $60 \%$ of projects is due to its budget and time overrun.

According to the survey conducted by [4], less than $40 \%$ of projects is considered to be successful and the main reason of failure is time and cost issues. There can be many reasons of project failure but big reason can be bad management.

According to [5], software requirements need to be prioritized before implementation especially when size of requirements is large. Prioritization is important phase during requirements management. There are four types of requirements. First type is process requirements which deals with time and cost issues of the projects from management perspective. Another type is business requirements that deals with benefits and advantages that are associated with the software. Third type is system requirements that are functional requirements and nonfunctional requirements. Functional requirements are system core requirements that system must do and must consist of while non-functional requirements define the quality attributes of the system. Many authors suggested techniques for prioritizing different types of requirements [6][7][8][9]. Prioritization is important for successful implementation and delivery of projects and in this context effective management plays important role.

In global software development (GSD) when vendors and clients are far away from each other's, due to barriers and challenges such as culture difference, lack of effective communication and coordination, gathering and managing of requirements become difficult [10][11]. GSD needs more attention as compare to local development. For effective requirements management and implementation, we need some success factors such as global project management tools, new and modern tools and technologies etc. [12].

\section{RESEARCH QUESTIONS}

The work presented in this paper is based on the following six research questions:

RQ1: What are the factors as identified from literature that have positive impact during requirement management phase of RE that can lead to timely delivery of software project?

RQ2: What are the factors in real practice that have positive impact during requirement management phase of RE that can lead to timely delivery of software project?

RQ3: What are those negative factors of requirements management as identified from literature that become the reason of software projects failure?

RQ4: What are those negative factors of requirements management in real practice that become the reason of software projects failure?

RQ5: Are there differences between the factors identified through the literature and the real-world practice?
RQ6: How much RMM is successful for the achieving of research objectives?

Through SLR, RQ1 and RQ3 will be answered while questionnaire survey/ Interviews will be conducted in software industries to answer RQ2 and RQ4.

\section{RESEARCH METHODOLOGY}

The methodology of the RMM consists of the following three phases.

Phase\#1: SLR will be conducted for data collection.

Phase\#2: Empirical studies will be conducted to validate the result of SLR and to find the practices for the mentioned factors.

Phase\#3: For evaluation and validation of RMM, Case study will be conducted. To explain the aforesaid three phases the following subsections are added.

RMM development life cycle is shown in figure 1 .

\subsection{Collection of data and its analysis}

i. CSFs (critical success factors): Factors that have a positive impact during requirement management.

ii. CRs (critical Risks): Factors that have negative impact during requirement management phase of RE that can lead to timely delivery of software project.

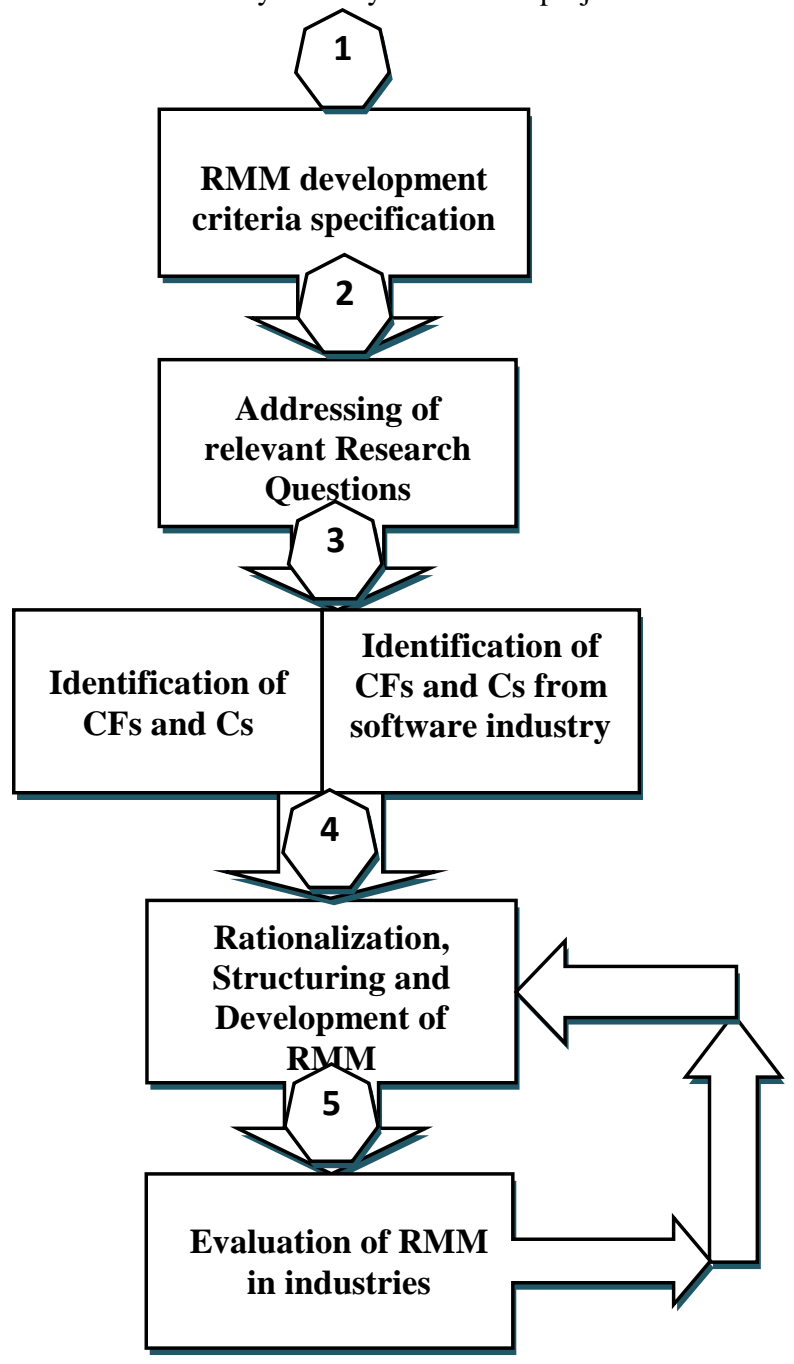

Figure 1: RMM Development Cycle Activities 
iii. Practices: For implementing CSFs, practices will be extracted and used.

SLR will be used to identify factors (CSFs and Cs). Through SLR we will extract, analyze and will explore data relevant to our research questions. SLR is different from ordinary literature reviews being formally planned and more systematic [13]. Many authors from literature used SLR successfully for retrieving factors in their relevant fields. Through SLR, it will be possible to identify factors from all relevant research. After retrieving, the identified factors will be analyzed from different perspectives such as size of requirements, company size, software type etc. because the reasons of success and failures can vary for different nature of requirements. To successfully implement factors (CSFs and Cs), a questionnaire survey will be conducted with experts working in the software industry. The purpose of this survey is:

- Validation of the results of SLR.

- $\quad$ To find new factors (CSFs and Cs) which are not previously identified.

- To identify practices for the success implementation of (CSFs and Cs).

After the design of RMM, one or more case studies will be conducted to evaluate the model. Any positive and negative feedback from software industries will be welcomed. Minimum five case studies will be conducted for validation of RMM.

\subsection{Development of RMM}

For the design of RMM we have used the five stages as shown in Figure 2. A similar approach has also been used by another researcher [14][15].

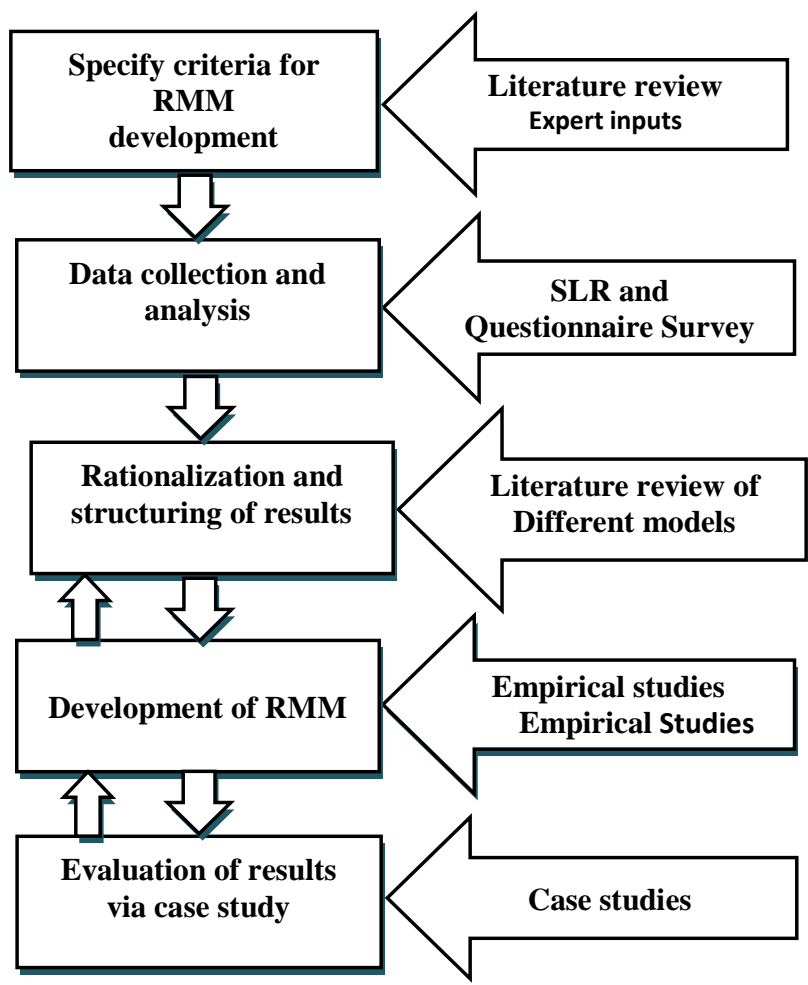

Figure 2: RMM development stages
The below mentioned two criteria will be used for the development and assessment of RMM.

A. User satisfaction: This criteria focus on the satisfaction of end user from the result of RMM. He / She should be able to use the RMM without any confusion or ambiguity to promote objectives according to their requirements and assumptions.

B.Usability: This measures of RMM stress on the organisation easiness. It describe that the structure of the RMM should be flexible and easy for understanding as organizations hesitate to accept models of complex nature and standards which require resources, training and efforts.

Data collection and analysis is the stage 2. Rationalization and structuring of results will be performed in stage 3 . Development of RMM based on the results of empirical studies in stage 4. Evaluation and validation of the RMM via case studies will be done in stage 5 .

\subsection{RMM structure}

We will build the structure of the RMM on the bases of following three extensions.

\section{- $\quad$ RMM levels}

- $\quad$ Factors (CSFs, Cs) in each level

- Practices and solutions for the implementation of factors

Classification of CSFs and Cs in different categories will be the base for defining the levels of RMM. Each level will be consisting of different factors (CSFs and Cs). For each factor in the particular level, practices will be given for its proper implementation. Like CMMI and other models, for organizations to achieve certain level they must address and should follow the practices for each CSFs and Cs under that particular level.

Figure 2 shows step by step detail activities of RMM development. Through Literature review of different models and from expert opinions, the criteria and design structure of RMM will be finalized.

Planned structure of the RMM is shown in Figure 3 . Relationship between RMM levels is also shown.

\subsection{RMM evaluation}

RMM will be validated through industrial case studies. For case studies maximum of five organizations will be selected. Case study is the best tool for evaluating any model in real environment. A focus group session will be arranged to get feedback from the participant about RMM. The criteria will be ease of use and user satisfaction as discussed in section 4.2. In focus group evaluation two or more people interact and generate ideas without getting help from researcher. Focus group is more open as compared to individual interview. 


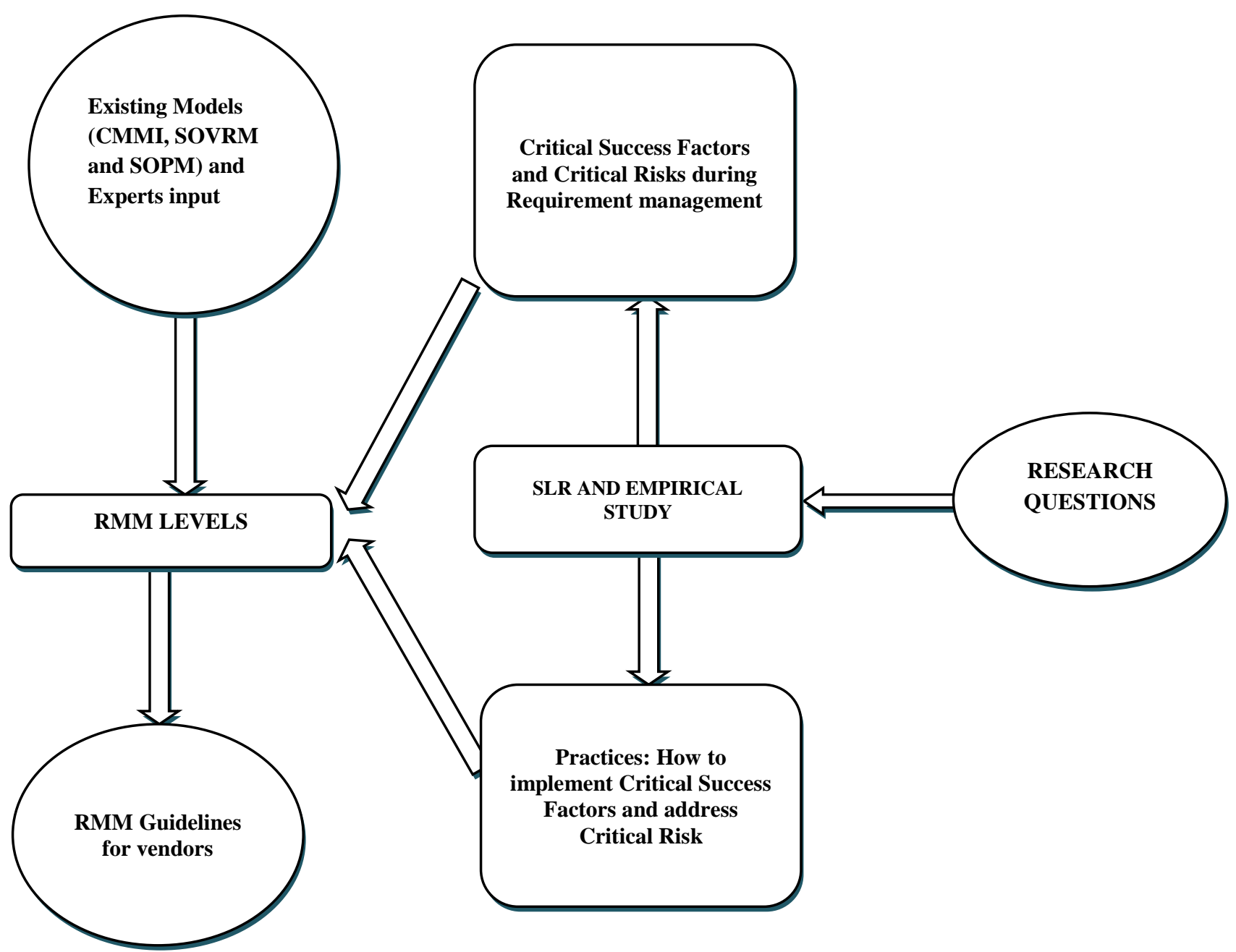

Figure 3: RMM Structure

\section{RESEARCH CARRIED UPTO DATE}

We have done the following research work so far:

- Identification of problem and objectives

- Research questions specification

- $\quad$ Selection of research methodology

- $\quad$ Defining structure of RMM

- Evaluation method selection

- Conduction of SLR

\section{CONCLUSION AND FUTURE WORK}

In our paper we have presented the structure of RMM with different levels and phases. We have discussed how this model will help vendors in better implementation of success factors during management. Detail methodology for the development of RMM was introduced. This model will be used as tool for software developers and will produce different assessment reports in different situations.

\section{REFERENCES}

[1] M. Yaseen, R. Naseem, Z. Ali, and G. Ullah, 'Identification Of Challenges During Requirements Implementation In Global Software Development: A Systematic', vol. 4, no. 1, pp. 23-40, 2019.
[2] M. Kuutila, M. Mäntylä, U. Farooq, and M. Claes, 'Time Pressure in Software Engineering: A Systematic Literature Review', 2017.

[3] H. Taherdoost and A. Keshavarzsaleh, 'A Theoretical Review on IT Project Success / Failure Factors and Evaluating the Associated Risks', 4th Int. Conf. Telecommun. Informatics, Sliema, Malta, no. August, pp. 80-88, 2015.

[4] H. Taherdoost and K. Abolfazl, 'Managing successful IT project; marketing perspective', Wseas.Us, vol. 28, no. 11, pp. 144-153, 2015

[5] M. Pergher and B. Rossi, 'Requirements prioritization in software engineering: A systematic mapping study', 2013 3rd Int. Work. Empir. Requir. Eng. Emp. 2013 Proc., pp. 40-44, 2013.

[6] R. Beg, R. P. Verma, and A. Joshi, 'Reduction in number of comparisons for requirement prioritization using B Tree', no. March, pp. 6-7, 2009.

[7] B. B. Jawale, G. K. Patnaik, and A. T. Bhole, 'Requirement Prioritization using Adaptive Fuzzy Hierarchical Cumulative Voting', 2017.

[8] R. Prioritization and U. Hierarchical, 'Requirements Prioritization Using Hierarchical Dependencies', pp. 459-464, 2018 
[9] N. Setiani and T. Dirgahayu, 'Clustering Technique for Information Requirement Prioritization in Specific CMSs', 2016.

[10] M. Yaseen, S. Baseer, and S. Sherin, 'Critical Challenges for Requirement Implementation in Context of Global Software Development: A Systematic Literature Review', pp. 120-125, 2015.

[11] M. Yaseen and Z. Ali, 'Success Factors during Requirements Implementation in Global Software Development: A Systematic Literature Review', vol. 8, no. 3, pp. 56-68, 2019.

[12] Z. Ali and M. Yaseen, 'Critical Challenges for Requirement Implementation in Global Software Development: A Systematic Literature Review Protocol with Preliminary Results', vol. 182, no. 48, pp. 17-23, 2019.
[13] M. Yaseen, S. Ali, Abdullah, N. Ullah “An Improved Framework for Requirement Implementation in the context of Global Software Development: A Systematic Literature Review Protocol", International Journal of Database Theory and Application Vol.9, No.6 (2016), pp.161-170.

[14] M. . Yaseen, S. . Baseer, S. . Ali, S. U. . Khan, and Abdullahb, 'Requirement implementation model (RIM) in the context of global software development', 2015 Int. Conf. Inf. Commun. Technol. ICICT 2015, 2015.

[15] M. Yaseen and U. Farooq, 'Requirement Elicitation Model (REM) in the Context of Global Software Development', Glob. J. Comput. Sci. Technol., vol. 7, no. 3, pp. 303-308, 2018. 